\title{
First-principles calculations of the self-trapped exciton in crystalline $\mathrm{NaCl}$
}

\author{
Vasili Perebeinos and Philip B. Allen \\ Department of Physics and Astronomy, State University of New York, Stony Brook, NY 11794-3800 \\ M. Weinert \\ Department of Physics, Brookhaven National Laboratory, Upton, NY 11973-5000
}

(November 13, 2018)

\begin{abstract}
The atomic and electronic structure of the lowest triplet state of the off-center $\left(\mathrm{C}_{2 \mathrm{v}}\right.$ symmetry) selftrapped exciton (STE) in crystalline $\mathrm{NaCl}$ is calculated using the local-spin-density (LSDA) approximation. In addition, the Franck-Condon broadening of the luminescence peak and the $\mathrm{a}_{1 g} \rightarrow \mathrm{b}_{3 u}$ absorption peak are calculated and compared to experiment. LSDA accurately predicts transition energies if the initial and final states are both localized or delocalized, but $1 \mathrm{eV}$ discrepancies with experiment occur if one state is localized and the other is delocalized.
\end{abstract}

71.35.Aa, 61.82.Ms, 78.20.Bh, 71.20.Ps

Unlike a molecule, an extended system such as a solid can support both spatially localized and delocalized single particle states and excitations. Physical properties such as luminescence can differ dramatically depending on which type of state occurs. Deciding theoretically whether a localized or delocalized solution exists is a challenging problem [1]. Here we examine $\mathrm{NaCl}$, a classic example [2] where electronic excitations self-localize by coupling to the lattice, creating local lattice distortions. Because the degree of localization will affect the Coulomb energies, approaches that incompletely cancel the self-interaction contribution to the exchange energy (e.g., the local density approximation) sometimes fail to predict the actual localized solution.

The ground state of alkali halides with one electron removed is the $\mathrm{V}_{K}$ center [2,3]: the resulting hole does not delocalize at the top of the valence band, but rather (symmetrically) attracts two $\mathrm{Cl}^{-}$ions into a tightly bound molecule [国:5], effectively becoming a $\mathrm{Cl}_{2}^{-}$molecular ion. The local symmetry of this atomic configuration is $\mathrm{D}_{2 \mathrm{~h}}$. An excess electron in bulk $\mathrm{NaCl}$ forms a large mobile (Fröhlich) polaron [6], but in the presence of a selftrapped hole forms a self-trapped exciton (STE). It has been suggested [7] that the self-trapped exciton state breaks symmetry and sits off-center with $\mathrm{C}_{2 \mathrm{v}}$ symmetry.

Although there have been many theoretical studies 1711] of the self-trapped exciton and $\mathrm{V}_{K}$ center problems, no density-functional calculations have been reported. In this letter we report local-spin-density approximation (LSDA) calculations of the STE and $\mathrm{V}_{K}$ center. The advantage of LSDA calculations is that they provide one of the simplest tools capable of providing a realistic model of this competition. In both cases, our LSDA calculations give undistorted, delocalized solutions with lower energy than the self-trapped solution, contrary to experiment. For the $\mathrm{V}_{K}$ center, no metastable local minimum trapped solution was found; however, for the (neutral) STE we find locally metastable solutions, with the on-center STE $0.14 \mathrm{eV}$ higher in energy than the offcenter STE solution, which in turn is higher by $0.20 \mathrm{eV}$ than a free electron-hole pair. Even with this discrepancy in the total energy, the atomic positions for the STE solution (see Table i) are reasonable and agree well with Hartree-Fock second-order Møller-Plesset (MP2) perturbation theory [10]. We focus on the properties of the local minimum solution for the off-center STE, which provides a test of the ability of density functional methods to treat the strong coupling of electronic and lattice degrees of freedom, and calculate the spectral properties of excited states.

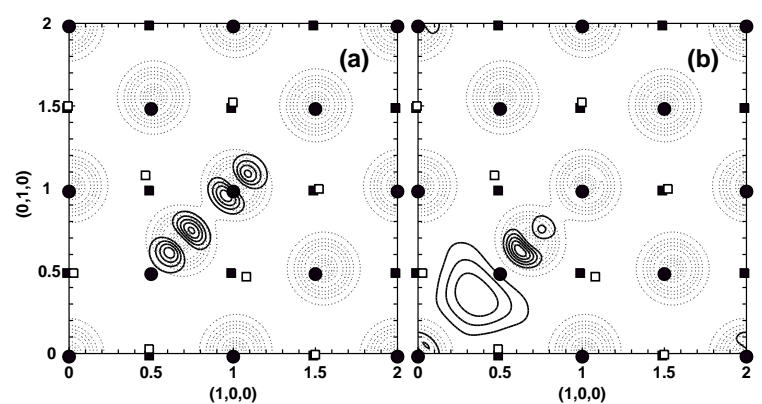

FIG. 1. The dotted contours show the total valence charge density in the STE. The solid contours show $|\Psi|^{2}$ for the trapped (a) hole and (b) electron. The open (filled) squares represent displaced (ideal) positions of the $\mathrm{Na}$ atoms; filled circles represent undisplaced $\mathrm{Cl}$ atoms. The lowest contours are 0.04 for the total charge, $0.01(0.002)$ for the hole (electron) state, with increments of $0.05,0.01$ and 0.002 e (a.u. $)^{-3}$, respectively.

To solve the LSDA equations we use a plane wave pseudopotential method 12 14 with a spin-dependent exchange-correlation potential [15], and full structural relaxation in a supercell approach. For most calculations, we used a 32 atom supercell with translation vectors $(2,0,0),(0,2,0)$ and $(1,1,1)$, giving a nearest neighbor distance between STEs of $9.4 \AA$, and used four special $\mathbf{k}$-points in the irreducible wedge for the Brillouin zone 
integrations. Tests varying the number of $\mathbf{k}$-points and supercell size suggest that these parameters are adequate [16]. One of the in $x-y$ plane nearest $\mathrm{Cl}$ and two $\mathrm{Na}$ atoms are most displaced while the rest of the atoms move by a much smaller amount. Some calculated structural parameters for the STE are given in Table 1 .

The calculated atomic displacements from the ideal $\mathrm{NaCl}$ structure for the off-center STE are shown in Fig. 1. The $b_{3 u}$ hole state in the triplet STE is localized on the $\mathrm{Cl}_{2}^{-}$molecule (Fig. 11a), with nearly equal weight on the two $\mathrm{Cl}$ ions. The last spin up electron $\left(\mathrm{a}_{1 g}\right)$ is mostly localized on the $(1 / 2,1 / 2,0)$ vacant halogen site (Fig. 1 $1 \mathrm{~b})$, as in the case of the F-center. The formation of the $\mathrm{Cl}_{2}^{-}$ "molecule" in the STE is mainly due to the shift of a single Cl. This asymmetric shift can be rationalized by noting that the Madelung energy (with canonical charges of \pm 1 for $\mathrm{Na}$ and $\mathrm{Cl}$ ) of the $\mathrm{D}_{2 \mathrm{~h}}$ configuration is about $0.17 \mathrm{eV}$ higher than the $\mathrm{C}_{2 \mathrm{v}}$ one, i.e., the ionic Madelung terms that favor the rock salt structure in the first place favor keeping one of the $\mathrm{Cl}$ ions on a lattice site; in addition, the extra electron in the STE (compared to the $V_{K}$ center) can lower its energy by this distortion.

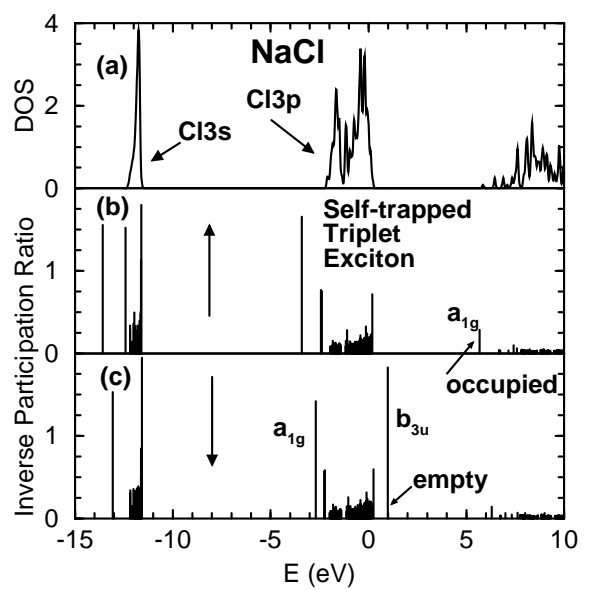

FIG. 2. (a) density of states (states/eV spin formula unit) of pure $\mathrm{NaCl}$ crystal, where the two spin orientations are equivalent. IPR of triplet STE for (b) spin up and (c) spin down electrons. The state labeled $b_{3 u}$ is empty and the state $\mathrm{a}_{1 g}$ is occupied in the STE. The $\mathrm{D}_{2 \mathrm{~h}}$ representations $\mathrm{b}_{3 u}$ and $\mathrm{a}_{1 g}$ became indistinguishable (both $\mathrm{A}_{1}$ ) in the true $\mathrm{C}_{2 \mathrm{v}}$ symmetry of the off-center state.

To obtain vibrational properties of the off-center STE, we made finite displacements from the equilibrium geometry. The $\mathrm{Cl}_{2}^{-}$stretching mode $\omega_{\text {str }}=242 \mathrm{~cm}^{-1}$ is smaller than the experimental Raman frequency $361 \mathrm{~cm}^{-1}$ [17]. ¿From the force matrix associated with the $\mathrm{Cl}_{2}^{-}$stretching mode, we found that only the two neighboring $\mathrm{Na}$ ions at $\left(\frac{1}{2}, 1,0\right)$ and $\left(1, \frac{1}{2}, 0\right)$ couple significantly. Unlike $\mathrm{H}$ center calculations 18], coupling to the $\mathrm{Na}$ atoms yields only a small shift in the frequency of the $\mathrm{Cl}_{2}^{-}$mode to $\omega_{\text {str }}=234 \mathrm{~cm}^{-1}$. Since the LSDA places the STE too high in energy, it is not too surprising that the curvature of the STE local minimum is underestimated,

Figure 2 shows the computed density of states (DOS) of the perfect $\mathrm{NaCl}$ and the inverse participation ratio $\left(\mathrm{IPR}=\mathrm{a}_{0}^{3} / 8 \int\left|\Psi_{i}(\vec{r})\right|^{4} d^{3} r\right)$ for states of majority and minority spin for the off-center STE. (The IPR is a measure of the localization of a state.) The localized hole $\mathrm{b}_{3 u}$ and electron $\mathrm{a}_{1 \mathrm{~g}}$ states lie in a gap of about $6 \mathrm{eV}$ between the conduction and valence bands of the perfect crystal, where as usual the LSDA underestimates the gap. Rather than using the single-particle eigenvalues, we obtain estimates of the excitation energies as the difference between total energies of different electronic configurations. To calculate the energy to create a free electron-hole pair (the gap energy), we occupy spin up states with one extra electron, while spin down states have one empty state. The energy difference between the two solutions $(6.44 \mathrm{eV})$ for the same atomic positions should correspond to the free electron-hole pair (experimental value $7.96 \mathrm{eV}$ [19]).

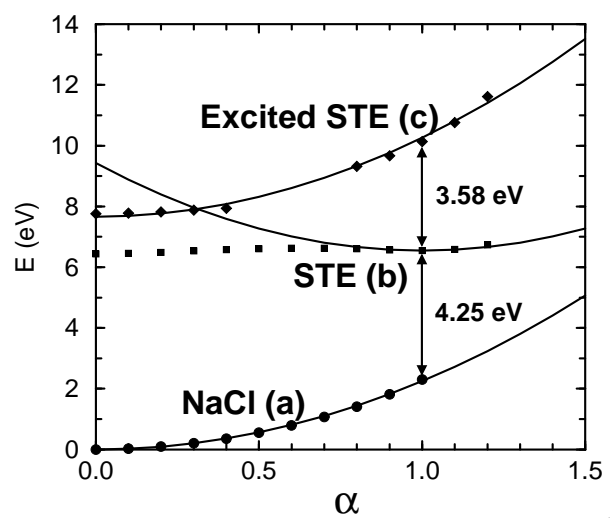

FIG. 3. Potential curves for pure $\mathrm{NaCl}$ (a), self-trapped exciton in the ground state (b) and electronically excited state $\left(\mathrm{a}_{1 g} \rightarrow \mathrm{b}_{3 u}\right)$ (c). The coordinate $\alpha$ measures the magnitude of the displacement, such that $\alpha=0$ and $\alpha=1$ correspond to the undisplaced $\mathrm{NaCl}$ crystal and the relaxed STE atomic configurations, respectively.

In the distorted STE solution, the energy of electronhole pair recombination was found by comparing energies of the STE solution with the energy of $\mathrm{NaCl}$ having the same atomic displacements as that of the STE. This energy of $4.25 \mathrm{eV}$ is roughly the same as the energy difference between $\mathrm{a}_{1 g}$ and $\mathrm{b}_{3 u}$ states given by the DOS, and compares to a value of $3.35 \mathrm{eV}$ obtained from a luminescence experiment [20] at $11 \mathrm{~K}$. The distorted $\mathrm{NaCl}$, with a lattice distortion energy of $2.5 \mathrm{eV}$ relative to the ideal $\mathrm{NaCl}$ positions, is in a highly excited vibrational state. Thus, significant Franck-Condon effects in the spectral properties of the STE are expected. To qualitatively describe the luminescence, a ground state potential curve was calculated for the configuration coordinate $\alpha$. It was assumed that all atoms move back to the perfect crystal positions proportionally to their distortions in the STE solution. The result is shown in Fig. 3, along with a quadratic fit $k_{a} \alpha^{2} / 2$ with one adjustable 
parameter $k_{a}$. The effective one-dimensional Schrödinger equation for $\Psi(\alpha)$ describes a harmonic oscillator with frequency $\omega_{a}=\sqrt{k_{a} / I}=109 \mathrm{~cm}^{-1}$. The moment of inertia $I=\sum M_{n} \delta \vec{R}_{n}^{2}$ was chosen so that $I \dot{\alpha}^{2} / 2$ equals the kinetic energy of the atoms with mass $M_{n}$, when they move from the initial displacements $\delta \overrightarrow{R_{n}}$.

The same type of the potential energy curve was calculated for the STE. The quadratic fit works only in the close vicinity of the exciton metastable minimum. The resulting frequency is $\omega_{b}=123 \mathrm{~cm}^{-1}$. The experimental 20] temperature $\mathrm{T}=11 \mathrm{~K}$ justifies a zero temperature approximation (the STE initial state is the vibrational ground state). Since the luminescence peak position corresponds to the vibrational level $n \approx 170$ of the electronic ground state, quasiclassical wavefunctions were used in the numerical integral evaluation. The sequence of vibrational sidebands should be replaced by a sequence of convolved densities of phonon states $D(\omega)$. We approximate this by a Gaussian, $D(\omega) \rightarrow \exp \left(-\omega^{2} / 2 \gamma^{2}\right) / \sqrt{2 \pi} \gamma$ with the width $\gamma=43 \mathrm{~cm}^{-1}$ chosen such that the first three moments coincide with the experimental [21] phonon DOS. This gives a luminescence width of $0.43 \mathrm{eV}$, while the experimental width is $0.63 \mathrm{eV}$.

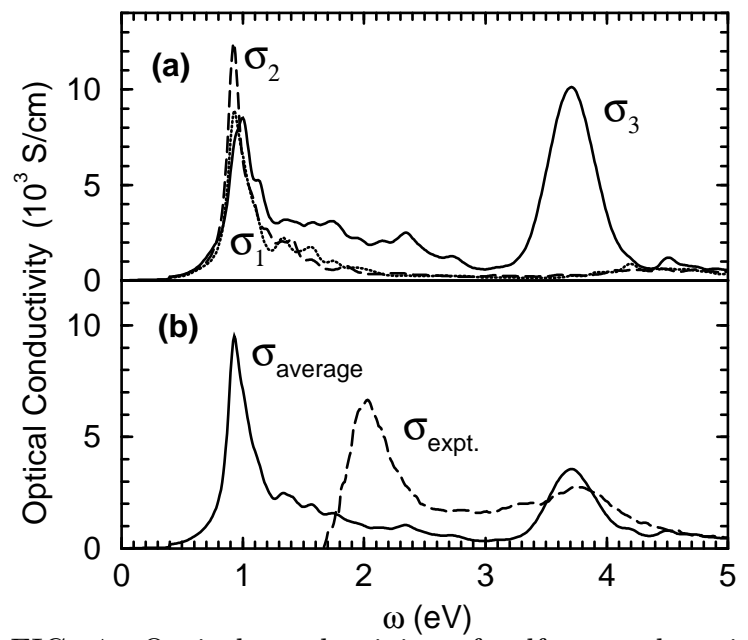

FIG. 4. Optical conductivity of self-trapped exciton in $\mathrm{NaCl}$ normalized per volume of a single exciton $a_{0}^{3} / 2$. (a) $\sigma_{1}(\omega): \vec{E} \perp$ to the molecular axis in the $x-y$ plane; $\sigma_{2}(\omega): \vec{E}$ along the $z$ direction; and $\sigma_{3}(\omega): \vec{E}$ along the $\mathrm{Cl}_{2}^{-}$molecule. (b): average conductivity compared with experiment 22 .

The optical response $\sigma(\omega)$ of the long-lived triplet STE also has been measured [22,23]. The diagonal part of the optical conductivity tensor is:

$$
\begin{gathered}
\sigma_{\alpha}(\omega)=\frac{\pi e^{2} N}{m^{2} \omega \Omega} \sum_{\ell \neq \ell^{\prime}} \sum_{\vec{k}}\left(f_{\ell \vec{k}}-f_{\ell^{\prime} \vec{k}}\right) \\
\left|\left\langle\ell \vec{k}\left|p_{\alpha}\right| \ell^{\prime} \vec{k}\right\rangle\right|^{2} \delta\left(\hbar \omega-E_{\ell^{\prime} \vec{k}}+E_{\ell \vec{k}}\right)
\end{gathered}
$$

where $f_{\ell \vec{k}}$ is the occupancy of the state $|\ell \vec{k}\rangle$. The spin state index is included in the band index $\ell$. Integration over the zone has been performed using $26 \mathrm{k}$-points in the

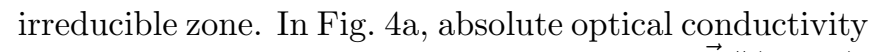
curves are shown for the three polarizations, $\vec{E} \|(1,-1,0)$, $(0,0,1)$, and $(1,1,0)$ (parallel to the the $\mathrm{Cl}_{2}^{-}$molecular axis). Fig. [ab shows the average conductivity $\sigma(\omega)=$ $\sum_{i} \sigma_{i}(\omega) / 3$, which is compared with experiment [22,23], rescaled so that the total weights under the both curves are the same.

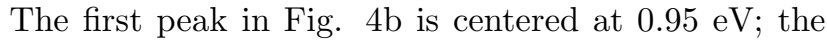
splitting between the peaks for the three different polarizations is not resolved in the calculations. Most of the weight in these peaks comes from transitions of the last localized spin-up $\mathrm{a}_{1 g}$ electron into empty conduction band delocalized states (see Fig. 2b).

The second peak centered at $3.58 \mathrm{eV}$ is the $\mathrm{a}_{1 g} \rightarrow \mathrm{b}_{3 u}$ transition for the spin down electron (see Fig. 2c). The energy difference between the ground state and electronically excited exciton state with the same atomic configuration turns out to be the same as the eigenvalue difference of $\mathrm{b}_{3 u}$ and $\mathrm{a}_{1 g}$ states of the ground STE. The excited exciton will lower its energy by moving atoms back to the undistorted positions of perfect $\mathrm{NaCl}$. To apply the Franck-Condon principle, we repeat the same type of calculations for the excited exciton as we did for $\mathrm{NaCl}$ and the ground state STE (Fig. 3). When two $\mathrm{Cl}$ atoms move away from each other, the $\mathrm{a}_{1 g}$ empty state merges with the valence $\mathrm{Cl} 3 p$ band, which makes it very difficult to choose which state to depopulate during the iterations. Instead we used the results for the ground state STE to obtain the energy of the electronically excited state by adding eigenvalue difference $\lambda_{\ell 1}-\lambda_{\ell 2}$ between two states for which dipole matrix element $\left|\left\langle\ell 1\left|p_{3}\right| \ell 2\right\rangle\right|^{2}$ is the largest. Results are shown on Fig. 3 along with a quadratic fit $\left(\omega_{c}=116 \mathrm{~cm}^{-1}\right)$ which works for the entire range of the parameter $\alpha$. The delta-function of Eq. (11) corresponding to the $\mathrm{a}_{1 g} \rightarrow \mathrm{b}_{3 u}$ transition was replaced by a sequence of convolved Gaussian peaks.

The transition energy $(3.58 \mathrm{eV})$ between the two localized states $\mathrm{a}_{1 g} \rightarrow \mathrm{b}_{3 u}$ (see Fig. 20 agrees well with the experimental peak at $3.8 \mathrm{eV}$. But when initial and final states have different degrees of localization, errors of an $\mathrm{eV}$ in the luminescence (LSDA: $4.25 \mathrm{eV}$, expt. 20]: 3.35 $\mathrm{eV}$ ) and in the optical excitation of the bound electron into the conduction band (LSDA: $0.95 \mathrm{eV}$, expt. [23]: 1.95, 2.13 and $2.00 \mathrm{eV}$ for three different field polarizations) occur. A possible explanation for this error is that in LSDA the incomplete cancellation of the large repulsive self-interaction energy $\int d \vec{r} d \overrightarrow{r^{\prime}} n_{i}(\vec{r}) n_{i}\left(\overrightarrow{r^{\prime}}\right) /\left|\vec{r}-\overrightarrow{r^{\prime}}\right|$ of a localized state $n_{i}=\left|\Psi_{i}\right|^{2}$ and the corresponding exchange term may destabilize a localized solution in favor of a delocalized one. Corrections such as self-interaction corrections (SIC) [24] or $\mathrm{LDA}+\mathrm{U}$ [25] may reduce the error; in SIC, shifts in the energy of a state $i$ on the order of $\int d^{3} r n_{i}^{4 / 3}(\vec{r})$ [24] are expected. If the degrees of localization of the two states are similar, however, then the LSDA transition energies are reasonable. 
In summary, we have presented LSDA calculations for the STE in $\mathrm{NaCl}$, including the coupling between the lattice and electronic states. The off-center STE is found to be more stable than the on-center STE, but both are metastable compared to free electron-hole pairs. Both luminescence and optical conductivity, including vibrational Franck-Condon effects, were also calculated. The hole state in the off-center STE is found to be rather evenly split between the two $\mathrm{Cl}$ atoms, but the electron state is localized to the vacant site left by the shifted $\mathrm{Cl}$ ion. The density functional description of electronic transitions between localized states, such as the $\mathrm{a}_{1 g} \rightarrow \mathrm{b}_{3 u}$ absorption peak, agree well with experiment. For transitions between the localized and delocalized states, discrepancies of order $1 \mathrm{eV}$ with experiment arise. Although the LSDA can capture many features of the STE states, when a localized solution competes with a delocalized solution, the incomplete cancellation of the self-interaction may destabilize the localized solution. Given the usefulness of the LSDA method for unraveling complex materials, it is important to test and develop approaches that can treat localized and delocalized states on the same footing.

\section{ACKNOWLEDGMENTS}

We thank M. L. Cohen, G. W. Fernando, P. M. Johnson, S.G. Louie, W. E. Pickett for discussions. This work was supported in part by NSF Grant No. DMR-9725037 and by DOE Grant No. DE-AC-02-98CH10886.

[1] F. Mauri and R. Car, Phys. Rev. Lett. 75, 3166 (1995); X. Zhang and D. A. Drabold, Phys. Rev. Lett. 83, 5042 (1999).

[2] K. S. Song and R. T. Williams, Self-Trapped Excitons, 2 nd ed. (Springer, Berlin, 1996); R. T. Williams and K. S. Song, J. Phys. Chem. Solids 51, 679 (1990).

[3] T. G. Castner and W. Känzig, J. Phys. Chem. Solids 3, 178 (1957).

[4] M. N. Kabler, in Point Defects in Solids I, edited by J. H. Crawford, Jr and L. M. Slifkin (Plenum, NY, 1972), p. $327 \mathrm{ff}$.

[5] C. P. Slichter, Principles of Magnetic Resonance (Harper and Row, NY, 1963), ch. 7.

[6] J. T. Devreese, in Encyclopedia of Applied Physics vol. 14 (VCH Publishers, Inc. 1996), p. 383ff.

[7] R. T. Williams, K. S. Song, W. L. Faust, and C. H. Leung, Phys. Rev. B 33, 7232 (1986); K. S. Song, Rev. Solid State Sci. 5, 477 (1991).

[8] A. N. Jette, T. L. Gilbert and T. P. Das, Phys. Rev. 184, 884 (1969).
[9] A. L. Shluger, N. Itoh, V. E. Puchin, and E. N. Heifets, Phys. Rev. B 44, 1499 (1991); V. E. Puchin, A. L. Shluger, K. Tanimura, and N. Itoh, Phys. Rev. B 47, 6226 (1993).

[10] V. E. Puchin, A. L. Shluger, and N. Itoh, Phys. Rev. B 52, 6254 (1995).

[11] S. E. Derenzo and M. J. Weber, Nucl. Instr. Meth. A 422, 111 (1999).

[12] N. Chetty, M. Weinert, T. S. Rahman, and J. W. Davenport, Phys. Rev. B 52, 6313 (1995).

[13] The Na pseudopotentials include a nonlinear core correction. Plane wave cutoffs were $\mathrm{E}_{\mathrm{pw}}=22 \mathrm{Ry}$ for the wave functions and $20 \mathrm{Ry}$ for the pseudopotential truncation [M. Alatalo, et al., Phys. Rev. B 60, 7680 (1999)]; for $\mathrm{E}_{\mathrm{pw}}=30 \mathrm{Ry}$, energy differences between the distorted and undistorted STE agreed within $0.01 \mathrm{eV}$.

[14] For the isolated $\mathrm{Cl}_{2}^{-}$molecule, the $\mathrm{Cl}-\mathrm{Cl}$ distance is 2.58 A (Hartree-Fock value: $2.65 \AA$ [8]; no experimental data) and the vibrational frequency is $243 \mathrm{~cm}^{-1}$ (expt.: 247 $\left.\mathrm{cm}^{-1}\right)$. For bulk $\mathrm{NaCl}$, calculated lattice constant (5.43 $\AA$; expt.: $5.6 \AA$ ) and bulk modulus (31.9 GPa; expt.: $26.6 \mathrm{GPa})$, are typical of local density calculations [e.g., S. Froyen and M. L. Cohen, J. Phys. C 19, 2623 (1986)].

[15] D. M. Ceperley and B. J. Adler, Phys. Rev. Lett. 45, 566 (1980).

[16] The energy differences between 1, 4, 13 and 26 k-point meshes are 0.094, 0.043, $0.029 \mathrm{eV}$ (off-center STE), 0.326, 0.090, $0.023 \mathrm{eV}$ (on-center STE) and 0.511, 0.130, 0.014 $\mathrm{eV}$ (free electron-hole pair). In the text, energy differences between the different atomic configurations are given for $26 \mathbf{k}$-points. For the 32-atom supercell, the offcenter shift of the $\mathrm{Cl}_{2}^{-}$molecule is $0.77 \AA$ (MP2: $0.95 \AA$ ). For a 72-atom cell, with $14.1 \AA$ STE separation and one special k-point, Cl-Cl distance $(2.58 \AA)$, nearest neighbor Na displacements $(0.50 \AA)$, and energy difference $(0.12$ $\mathrm{eV})$ are essentially unchanged; the off-center shift $(0.84$ $\AA$ ) is slightly larger since the energy cost of $\mathrm{Cl}_{2}^{-}$displacements is small.

[17] K. Tanimura, T. Suzuki, and N. Itoh, Phys. Rev. Lett. 68, 635 (1992); T. Suzuki, K. Tanimura, and N. Itoh, Phys. Rev. B 49, 7233 (1994).

[18] A. Svane, E. A. Kotomin, and N. E. Christensen, Phys. Rev. B 53, 24 (1996).

[19] R. S. Knox and K. J. Teegarden, in Physics of Color Centers, edited by W. B. Fowler (Academic, NY, 1968), p. $625 \mathrm{ff}$.

[20] M. Ikezawa and T. Kojima, J. Phys. Soc. Japan 27, 1551 (1969).

[21] G. Raunio and S. Rolandson, Phys. Rev. B 2, 2098 (1970).

[22] R. T. Williams and M. N. Kabler, Phys. Rev. B 91897 (1974).

[23] K. Tanimura, in Defect Processes Induced by Electronic Excitation in Insulators, edited by N. Itoh (World Scientific, Singapore, 1989), p. 178.

[24] J. P. Perdew and A. Zunger, Phys. Rev. B 23, 5048 (1981); A. Svane and O. Gunnarsson, Phys. Rev. B 37, 9919 (1988); M. R. Pederson and C. C. Lin, J. Chem. Phys. 88, 1807 (1988).

[25] A. I. Liechtenstein, V. I. Anisimov and J. Zaanen, Phys. Rev. B. 52, R5468 (1995); A. B. Shick, A. I. Liechten- 
stein, W. E. Pickett, Phys. Rev. B 60, 10763 (1999).

TABLE I. The equilibrium Cl-Cl distance $r_{C l-C l}$, displacements of the nearest Na ions $\Delta_{N a}$ in $(\AA)$, for the STE. Present results are compared to the Hartree-Fock (MP2) theory 10 .

\begin{tabular}{lcc}
\hline \hline Configuration & $r_{C l-C l}(\mathrm{MP} 2)$ & $\Delta_{N a}(\mathrm{MP} 2)$ \\
\hline $\mathrm{STE}_{2 \mathrm{~h}}$ & $2.73(2.654)$ & $0.35(0.383)$ \\
STE $\mathrm{C}_{2 \mathrm{v}}$ & $2.59(2.525)$ & $0.51(0.488)$ \\
\hline \hline
\end{tabular}

